\title{
Safety and Recipient Satisfaction of Propofol Sedation in Outpatient Endoscopy: A 24-Hour Prospective Investigation Using a Questionnaire Survey
}

\author{
Yoshihide Kanno, Tetsuya Ohira, Yoshihiro Harada, Shinsuke Koshita, Takahisa Ogawa, Hiroaki Kusunose, Yoshiki Koike, Taku \\ Yamagata, Toshitaka Sakai, Kaori Masu, Keisuke Yonamine, Kazuaki Miyamoto, Megumi Tanaka, Tomohiro Shimada, Fumisato \\ Kozakai, Kazuki Endo, Haruka Okano, Daichi Komabayashi, Takeshi Shimizu, Shohei Suzuki and Kei Ito \\ Department of Gastroenterology, Sendai City Medical Center, Sendai, Japan
}

Background/Aims: The aim of this study was to evaluate the safety of sedation with propofol as an alternative to benzodiazepine drugs in outpatient endoscopy.

Methods: In this prospective study, examinees who underwent outpatient endoscopy under propofol sedation and submitted a nextday questionnaire with providing informed consent were evaluated. Periprocedural acute responses, late adverse events within 24 hours, and examinee satisfaction were evaluated.

Results: Among the 4,122 patients who received propofol in the 17,978 outpatient-based endoscopic examinations performed between November 2016 and March 2018, 2,305 eligible examinees (esophagogastroduodenoscopy for 1,340, endoscopic ultrasonography for 945 , and total colonoscopy for 20 ) were enrolled, and their responses to a questionnaire were analyzed. The mean propofol dose was $69.6 \pm 24.4 \mathrm{mg}$ (range, $20-200 \mathrm{mg}$ ). Diazepam, midazolam, and/or pentazocine in combination with propofol was administered to 146 examinees. Mild oxygen desaturation was observed in 59 examinees (2.6\%); and mild bradycardia, in $2(0.09 \%)$. Other severe reactions or late events did not occur. After eliminating 181 invalid responses, $97.7 \%(2,065 / 2,124)$ of the patients desired propofol sedation in future examinations.

Conclusions: Propofol sedation was found to be safe-without severe adverse events or accidents-for outpatient endoscopy on the basis of the patients' next-day self-evaluation. Given the high satisfaction level, propofol sedation might be an ideal tool for painless endoscopic screening. Clin Endosc 2021;54:340-347

Key Words: Cancer screening; Endoscopic ultrasonography; Esophagogastroduodenoscopy; Propofol; Sedation

\section{INTRODUCTION}

Endoscopists have sought methods enabling painless endoscopy to increase checkup and follow-up rates for detecting diseases at an early stage. Sedation is a useful option to realize this

Received: May 22, 2020 Revised: June 26, 2020

Accepted: July 6, 2020

Correspondence: Yoshihide Kanno

Department of Gastroenterology, Sendai City Medical Center, Tsurugaya 5-22-1, Miyagino-ku, Sendai, Miyagi 983-0824, Japan

Tel: +81-22-252-1111, Fax: +81-22-252-9431, E-mail: yoshi-hk@openhp.or.jp ORCID: https://orcid.org/0000-0002-2044-5483

(c) This is an Open Access article distributed under the terms of the Creative Commons Attribution Non-Commercial License (http://creativecommons.org/ licenses/by-nc/3.0) which permits unrestricted non-commercial use, distribution, and reproduction in any medium, provided the original work is properly cited. goal as long as safety is not compromised. Safety includes not only periprocedural acute responses but also delayed events that may occur after the examinees leave the hospital following outpatient-based examinations.

Benzodiazepine $(\mathrm{Bz})$ drugs have been commonly used for sedation worldwide. ${ }^{1}$ Although Bzs can be safely used owing to their relatively mild profiles and considerable use experience, a few concerns remain, including oversedation and long-lasting effects. Oversedation causes respiratory suppression, cardiovascular reaction, and prolonged recovery time. The long duration of the drug effects necessitates prolonged care and, thus, recovery beds. Moreover, residual effects such as drowsiness, impaired judgement, and physical instability might cause dangerous accidents. 
Propofol has been considered an ideal alternative because of its extremely rapid drug kinetics, reported by several previous publications confirming its clinical favorability. ${ }^{1-6}$ However, its safety has not been fully established for outpatient use because the safety of recipients after they have left the hospital has not been studied. The safety of sedatives should be verified in relation to an examinee's behavior outside the hospital to validate their general use in common practice. Therefore, we conducted a prospective study to evaluate the safety of propofol by using a questionnaire to which examinees replied 24 hours after endoscopy.

\section{MATERIALS AND METHODS}

\section{Participant enrollment}

All examinees who received propofol sedation for outpatient-based endoscopic examination, including esophagogastroduodenoscopy (EGD), endoscopic ultrasonography (EUS), and total colonoscopy (TCS), between November 2016 and March 2018 were recruited for this study. The decision to be sedated using propofol was made by the examinee voluntarily. Before obtaining informed consent for propofol use, the characteristics of propofol and Bzs (diazepam and midazolam) were impartially explained without a physician's recommendation or arbitrariness for propofol use if no medical reasons exist to recommend it. Propofol was not recommended for examinees with the following conditions: (1) age < 18 years; (2) pregnancy; (3) moderate or severe comorbidity of cardiopulmonary, renal, or liver function; (4) allergy or intolerance to propofol, chicken egg, soy, or coconut; and (5) no written informed consent for endoscopy and propofol use.

The exclusion criteria for study enrollment were as follows: (1) previous participation in the study, (2) endoscopy as a treatment intervention, (3) inability to respond and send a questionnaire, and (4) no written informed consent for participation in the study.

This prospective observational single-arm study was conducted at Sendai City Medical Center after approval by the institutional review board of Sendai City Medical Center.

\section{Procedures and monitoring}

A venous route was established using an indwelling catheter for all the examinees who requested for propofol sedation. Oxygen saturation levels and heart rates were monitored using a pulse oxymeter before, during, and after the procedure until full recovery of wakeful consciousness. Blood pressure was measured before the procedure and when needed during the procedure.
For EGD and EUS, $80 \mathrm{mg}$ of lidocaine was sprayed (10 pushes of $8 \%$ lidocaine) into the throat before sedation. For TCS, the examinees took $10 \mathrm{~mL}$ of $0.75 \%$ sodium picosulfate the night before and 2,000 $\mathrm{mL}$ of enterolavage liquid with polyethylene glycol the morning before the procedure. Immediately before inserting the colonoscope, $5 \mathrm{mg}$ of scopolamine butylbromide was intravenously administered.

Propofol was administered by an assisting nurse under the direction of an endoscopist who was experienced in sedation. An anesthesiologist was always present in the hospital during the examinations performed in this study. The initial amount of propofol administration was $60 \mathrm{mg}$ for examinees aged $<60$ years, $40 \mathrm{mg}$ for those aged between 60 and 79 years, and $20 \mathrm{mg}$ for those aged $\geq 80$ years. The initial amount was reduced at the endoscopist's discretion on the basis of comorbidity. When the sedation depth was insufficient, 10-20 mg of propofol was added if cardiopulmonary suppression was not problematic. The maximal dose was defined to be $200 \mathrm{mg}$. Diazepam, midazolam, and pentazocine were used in combination when propofol alone was insufficient.

When adverse events occurred owing to the endoscopic procedures or sedatives, the endoscopist took appropriate actions, including oxygen administration, drug administration, and/or cessation of endoscopy. For a persistently low level of oxygen saturation ( $<89 \%$ ), oxygen was administered.

For patients who underwent examinations under coverage by the Japanese national insurance system, the fee for propofol use was similarly covered under approval by the regional Bureau of Health and Welfare. When examinees had to bear the cost of the check-up, the costs for propofol sedation were covered by our institution.

After the endoscopic examination, the examinees were required to lie on a bed in a recovery room for at least 30 minutes. When a $\mathrm{Bz}$ drug or pentazocine was administered in combination, the examinees were confined for an additional 30 minutes. After the mandatory rest time, a nurse checked if the examinee could (1) clearly respond to the nurse and (2) stably walk, and (3) had a $\geq 94 \%$ oxygen saturation level. When all three conditions were satisfied, the examinee was permitted to leave the recovery room. In cases where the examinee was unstable on their feet or had a low oxygen saturation level before sedation, a physician judged if the drug reaction had sufficiently diminished. Otherwise, the examinee was required to rest until they fully recovered. Driving after the examination with sedation was prohibited on the day of the examination, although this is not illegal in Japan.

\section{Evaluated outcomes}

An attending nurse in the examination room and a nurse in 
the recovery room recorded the kinds and amounts of administered drugs, adverse events, and necessity for oxygen administration. A questionnaire was handed to the examinee after recovery. It was filled out $\geq 24$ hours after the examination and posted with the examinee's written consent. The questions were as follows:

(1) Until when did you feel drowsiness, sleepiness, or physical instability among the following options?

a. Before hospital discharge

b. Until early evening (around sunset) on the same day

c. Until nighttime on the same day

d. Until the next morning

e. Not sure

(2) Did any accident occur because of impaired judgment caused by the effects of the drug?

(3) Did you feel any symptoms other than drowsiness, sleepiness, and physical instability?

(4) Do you hope to receive propofol sedation in future endoscopic examinations?

a. Yes

b. Yes, but I desire deeper sedation

c. No, I desire another kind of sedative

d. No, I desire to receive endoscopy without sedation

e. Not sure

For evaluating factors related to outcomes, statistical calculations were conducted using the Fisher exact test or $t$-test if necessary.

\section{Ethical considerations}

Propofol use and this prospective investigation were approved by the institutional review board of Sendai City Medical Center (approval No. 20160027). This prospective study was registered in the University Hospital Medical Information Network (UMIN; issued ID No. UMIN000040330). As mentioned earlier, written informed consent for all the endoscopic examinations, sedation, propofol use, and participation in this study was obtained from all the participants.

\section{RESULTS}

In the 17,978 outpatient-based endoscopic examinations performed between November 2016 and March 2018, 4,122 examinees underwent endoscopic examination under propofol sedation and 2,317 were eligible to participate in this study and submitted the questionnaire with their written consent (Fig. 1). After eliminating 12 examinees who submitted invalid questionnaires with incomplete signatures or unreadable answers or who posted it on the procedure date, 2,305 examinees were included in the analyses. The ages, sexes, and types of endoscopy are shown in Table 1.

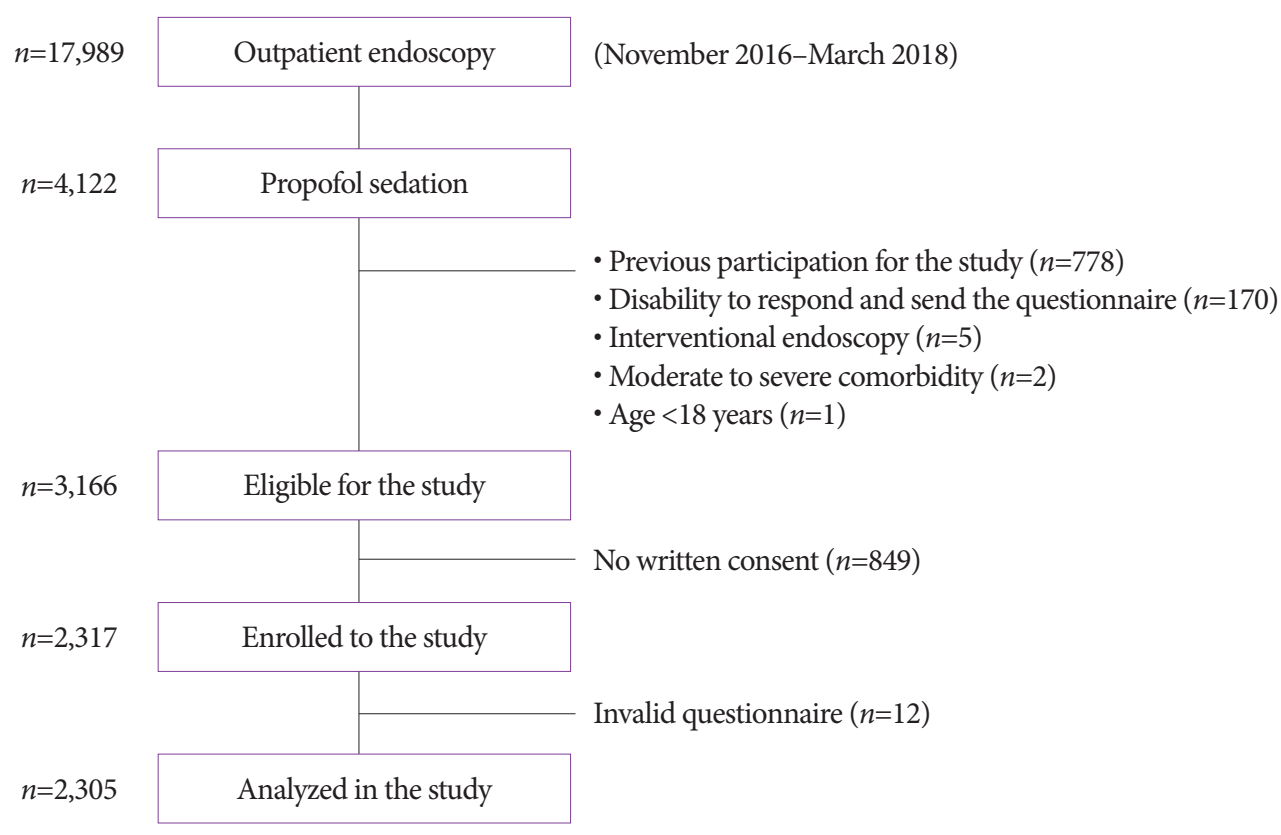

Fig. 1. Flowchart for participant enrollment. 
Table 1. Distribution of the Participants

\begin{tabular}{lc}
\hline & $\boldsymbol{n}=\mathbf{2 , 3 0 5}$ \\
\hline Age & \\
Mean \pm SD, yr & $64.3 \pm 11.5$ \\
Range, yr, $n$ (\%) & $23-91$ \\
$18-29$ & $6(0.3 \%)$ \\
$30-39$ & $49(2.1 \%)$ \\
$40-49$ & $226(9.8 \%)$ \\
$50-59$ & $448(19.4 \%)$ \\
$60-69$ & $736(31.9 \%)$ \\
$70-79$ & $687(29.8 \%)$ \\
$80-89$ & $151(6.6 \%)$ \\
$90-99$ & $2(0.1 \%)$ \\
Sex, $n$ (\%) & \\
Male & $1,215(52.7 \%)$ \\
Female & $1,090(47.3 \%)$ \\
Examination, $n$ (\%) & \\
EGD & $1,340(58.1 \%)$ \\
EUS & $945(41.0 \%)$ \\
TCS & $20(0.9 \%)$ \\
Examination time, min & \\
Overall, median (range) & $6(2-29)$ \\
EGD, median (range) & $5(2-10)$ \\
TCS, median (range) & $7(4-26)$ \\
\hline & $13(8-29)$ \\
\hline
\end{tabular}

EGD, esophagogastroduodenoscopy; EUS, endoscopic ultrasonography; SD, standard deviation; TCS, total colonoscopy.
The mean propofol dose was 69.6 $\pm 24.4 \mathrm{mg}$ (range, 20-200 $\mathrm{mg}$ ) and was combined with diazepam for 92 examinees, midazolam for 39, and/or pentazocine for 21 (Table 2). A drug combination was used more frequently for the examinees aged $<70$ years than for the older examinees ( $7.4 \%$ vs. $4.4 \%$, $p=0.005$ ).

Mild oxygen desaturation requiring oxygen administration at a rate of $2-3 \mathrm{~L} / \mathrm{min}$ occurred in 59 examinees (2.6\%), although no severe respiratory suppression occurred (Table 3 ). Oxygen desaturation frequently occurred in (1) the examinees to whom $\geq 170 \mathrm{mg}$ of propofol was administered (27.3\% [3/14] vs. $2.4 \%[56 / 2,291], p=0.005$; Table 4$)$, (2) elderly examinees aged $\geq 70$ years $(4.0 \%$ [34/840] vs. $1.7 \%[25 / 1,465], p=0.001)$, and (3) the examinees who received a combination of sedatives as compared with those who only received propofol (10.3\% [15/146] vs. $2.0 \%$ [44/2,159], $p<0.001)$.

Two examinees developed hypotension with mild bradycardia, which required administration of intravenous atropine sulfate $(0.09 \%)$, namely a 64 -year-old woman who underwent EUS with 60-mg propofol and an 84-year-old man who underwent EUS with 80-mg propofol (Table 3). A 78-year-old male EGD examinee who was sedated using 40-mg propofol and had been hospitalized owing to a feeling of illness after previous EGD examinations without propofol was hospitalized because of a similar feeling. A loose tooth fell out after EGD in the case of a 78-year-old woman who was sedated using 50-mg propofol. No other severe adverse events that

Table 2. Drug Dosage

\begin{tabular}{|c|c|c|c|c|}
\hline & $\begin{array}{c}\text { EGD } \\
(n=1,340)\end{array}$ & $\begin{array}{c}\text { EUS } \\
(n=945)\end{array}$ & $\begin{array}{c}\text { TCS } \\
(n=20)\end{array}$ & $\begin{array}{c}\text { Overall } \\
(n=2,305)\end{array}$ \\
\hline \multicolumn{5}{|l|}{ Propofol } \\
\hline Mean \pm SD, mg & $61.9 \pm 17.7$ & $80.7 \pm 28.2$ & $60.5 \pm 20.9$ & $69.6 \pm 24.4$ \\
\hline Range, mg & $20-160$ & $20-200$ & $30-100$ & $20-200$ \\
\hline $10-40$ & $234(17.5 \%)$ & $59(6.2 \%)$ & $6(30 \%)$ & $299(13.0 \%)$ \\
\hline $50-80$ & $1,000(74.6 \%)$ & $566(59.9 \%)$ & $11(55.0 \%)$ & $1,577(68.4 \%)$ \\
\hline $90-120$ & $100(7.5 \%)$ & $258(27.3 \%)$ & $3(15.0 \%)$ & $361(15.7 \%)$ \\
\hline $130-160$ & $6(0.4 \%)$ & $48(5.1 \%)$ & 0 & $54(2.3 \%)$ \\
\hline $170-200$ & 0 & $14(1.5 \%)$ & 0 & $14(0.6 \%)$ \\
\hline \multicolumn{5}{|l|}{ Combined medicine } \\
\hline Any & $29(2.2 \%)$ & $115(12.2 \%)$ & $2(10.0 \%)$ & $146(6.3 \%)$ \\
\hline $\begin{array}{l}\text { Diazepam } \\
(\text { mean } \pm S D \text {, range }[\mathrm{mg}])\end{array}$ & $\begin{array}{c}13(1.0 \%) \\
(4.8 \pm 0.7,2.5-5.0)\end{array}$ & $\begin{array}{c}78(8.3 \%) \\
(5.3 \pm 1.7,2.0-10.0)\end{array}$ & $\begin{array}{c}1(5.0 \%) \\
(2.0)^{\mathrm{a})}\end{array}$ & $\begin{array}{c}92(4.0 \%) \\
(5.2 \pm 1.6,2.0-10.0)\end{array}$ \\
\hline $\begin{array}{l}\text { Midazolam } \\
(\text { mean } \pm S D \text {, range }[\mathrm{mg}])\end{array}$ & $\begin{array}{c}6(0.4 \%) \\
(2.5 \pm 0.8,2.0-4.0)\end{array}$ & $\begin{array}{c}32(3.4 \%) \\
(3.8 \pm 1.9,2.0-10.0)\end{array}$ & $\begin{array}{c}1(5.0 \%) \\
(5.0)^{\mathrm{a})}\end{array}$ & $\begin{array}{c}39(1.7 \%) \\
(3.6 \pm 1.8,2.0-10.0)\end{array}$ \\
\hline $\begin{array}{l}\text { Pentazocine } \\
(\text { mean } \pm S D \text {, range }[\mathrm{mg}])\end{array}$ & $\begin{array}{c}10(0.7 \%) \\
(13.5 \pm 3.2,7.5-15.0)\end{array}$ & $\begin{array}{c}9(1.0 \%) \\
(11.7 \pm 4.0,7.5-15.0)\end{array}$ & $\begin{array}{c}2(10.0 \%) \\
(11.3 \pm 5.3,7.5-15.0)\end{array}$ & $\begin{array}{c}21(0.9 \%) \\
(12.5 \pm 3.6,7.5-15.0)\end{array}$ \\
\hline
\end{tabular}

${ }^{\text {a) }}$ Since the sample number is one, the $\mathrm{SD}$ and range are not demonstrated.

EGD, esophagogastroduodenoscopy; EUS, endoscopic ultrasonography; SD, standard deviation; TCS, total colonoscopy. 
Table 3. Periprocedural Acute Events

\begin{tabular}{lc}
\hline & $\boldsymbol{n}=\mathbf{2 , 3 0 5}$ \\
\hline Overall & $63(2.7 \%)$ \\
Mild oxygen desaturation & $59(2.6 \%)$ \\
Mild bradycardia & $2(0.1 \%)$ \\
Others $^{\text {a) }}$ & $2(0.1 \%)$ \\
\hline
\end{tabular}

a) The other 2 events were a feeling of illness and loss of a tooth.

Table 4. Details of the Distribution of the Examinees Who Required Oxygen Administration

\begin{tabular}{lc}
\hline & $\boldsymbol{n}=\mathbf{2 , 3 0 5}$ \\
\hline Overall & $59 / 2,305(2.6 \%)$ \\
Age, yr & \\
$<60$ & $8 / 729(1.1 \%)$ \\
$60-69$ & $17 / 736(2.4 \%)$ \\
$70-79$ & $29 / 687(4.4 \%)$ \\
$80-89$ & $5 / 151(3.4 \%)$ \\
$<90$ & $0 / 2(0.0 \%)$
\end{tabular}

Propofol, mg

$\begin{array}{lc}10-40 & 10 / 299(3.5 \%) \\ 50-80 & 35 / 1,577(2.3 \%) \\ 90-120 & 10 / 361(2.8 \%) \\ 130-160 & 1 / 54(1.9 \%) \\ 170-200 & 3 / 14(27.3 \%)\end{array}$

Drug combination

None

$44 / 2,159(2.0 \%)$

Combined (any drug)

$15 / 146(10.3 \%)$

Diazepam

$6 / 92(6.5 \%)$

Midazolam

$8 / 39(20.5 \%)$

Pentazocine

$3 / 21(14.3 \%)$

required medication, procedure cessation, mask ventilation, tracheal intubation, or chest compression occurred. Overall, the total rate of acute adverse events was $2.7 \%(63 / 2,305$, Table 3).

From the data compiled from the submitted questionnaires, the feelings of drowsiness, sleepiness, and physical instability disappeared before hospital discharge in $86.6 \%$ of the participants, whereas in $2.7 \%$ of the participants, these feelings persisted until nighttime on the same day or the next morning (Table 5). After eliminating the examinees who answered "not sure" or invalidly (by providing no answer or checking multiple options), the feelings disappeared prior to hospital dis- charge in $88.0 \%$ of the patients and by early evening in $97.3 \%$. Serious unfavorable events, including traffic accidents, falling, incidents related to amnesia, and abnormal behavior, were not reported.

Unfavorable symptoms after hospital discharge were reported by 151 examinees (6.6\%, Table 5). Miscellaneous symptoms were reported by 19 examinees (others in Question 3 in Table 5), including a feeling of illness, pyrexia (EGD with $60 \mathrm{mg}$ of propofol), judgment impairment (EGD with $70 \mathrm{mg}$ of propofol), head itchiness (EGD with $60 \mathrm{mg}$ of propofol), pain inside the nose (EGD with $40 \mathrm{mg}$ of propofol), mouth dryness (EGD with $70 \mathrm{mg}$ of propofol), coughing (EGD with $60 \mathrm{mg}$ of propofol), heartburn (EGD with $50 \mathrm{mg}$ of propofol), hand numbness (EGD with $40 \mathrm{mg}$ of propofol), leg cramps (EGD with $60 \mathrm{mg}$ of propofol), hallucination (EUS with 120 $\mathrm{mg}$ of propofol), tinnitus (EUS with $70 \mathrm{mg}$ of propofol), eye floater (EUS with $80 \mathrm{mg}$ of propofol), subepithelial bleeding on a lip (EUS with $80 \mathrm{mg}$ of propofol), body itchiness (EUS with $100 \mathrm{mg}$ of propofol), lumbar pain (EUS with $100 \mathrm{mg}$ of propofol), a feeling of poor intestinal peristalsis (EUS with $60 \mathrm{mg}$ of propofol), flatus (EUS with $60 \mathrm{mg}$ of propofol), and involuntary defecation (EUS with $70 \mathrm{mg}$ of propofol). Most symptoms such as throat pain/discomfort $(n=60)$, abdominal pain/discomfort $(n=10)$, and pain at the puncture site of the indwelling catheter $(n=3)$ did not seem to be directly linked to propofol use. All symptoms improved without medical intervention.

After eliminating 181 participants who answered "not sure" or invalidly, $97.7 \%(2,065 / 2,124)$ of the participants desired propofol sedation in future examinations. For each examination, $97.9 \%(1,238 / 1,264)$ of the participants who underwent EGD, 97.3\% (818/841) who underwent EUS, and 100\% (19/19) who underwent TCS replied to have such a preference.

No significant differences in all the items evaluated in the questionnaire survey were found between the examinees who received and did not receive combinations of diazepam, midazolam, and/or pentazocine (Table 5).

\section{DISCUSSION}

Propofol has several advantageous profiles that are useful in sedation such as rapid induction and its extremely short half-life as compared with the traditional Bz drugs. Recipients lose consciousness immediately after injection and recover consciousness a few minutes after the last injection. This characteristic appears ideal for the usual endoscopic examinations that require only 5-10 minutes. Psychomotor recovery evaluated using driving simulators has been reported with signifi- 


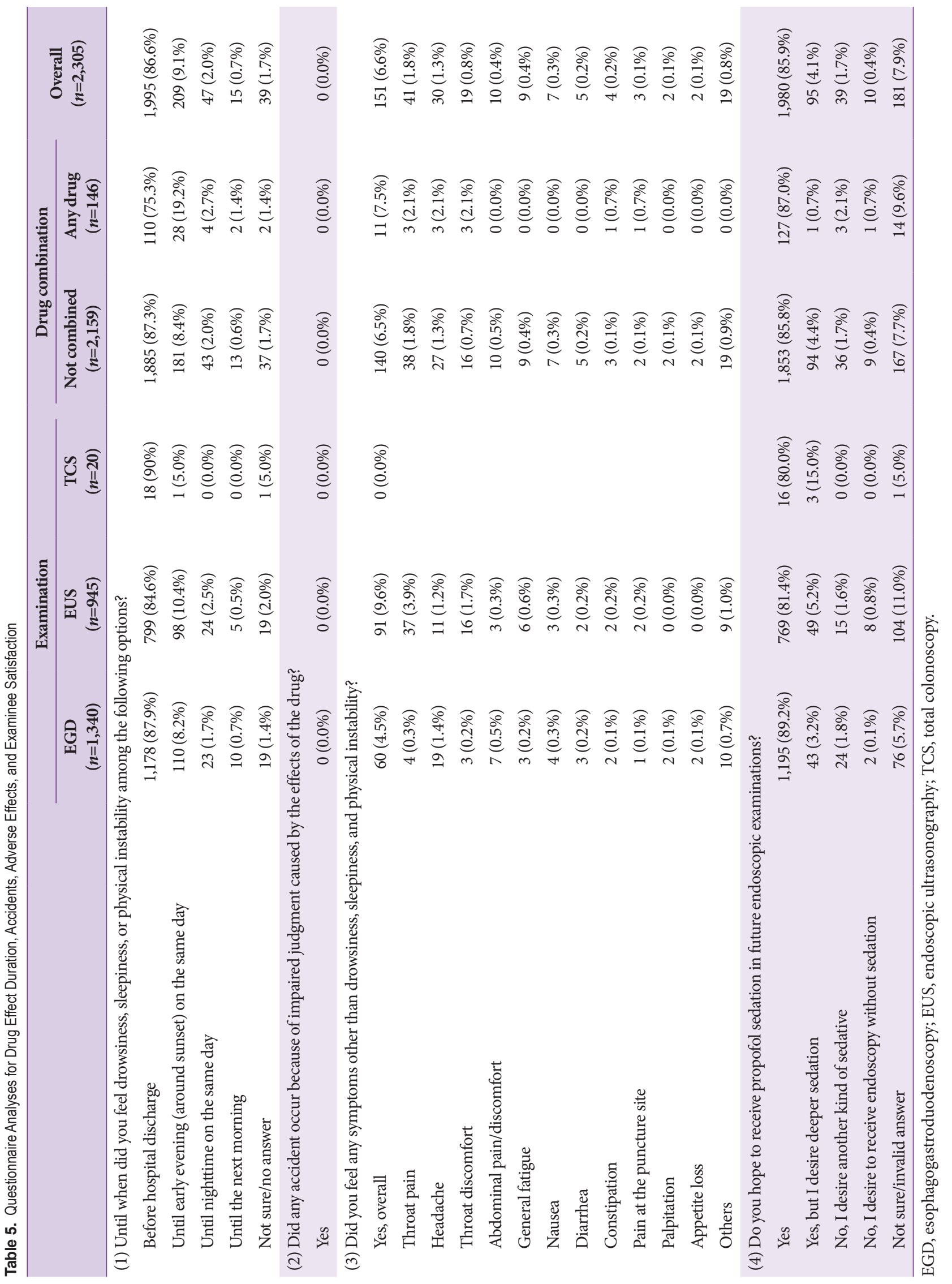


cantly favorable outcomes in comparison with the traditional sedatives in several studies. ${ }^{2-7}$

In a meta-analysis published in 2019,11 randomized controlled trials involving 1,767 recipients were analyzed to compare the outcomes of propofol with those of Bzs as sedatives for endoscopy. ${ }^{1}$ Although Bzs appear to lead to oxygen desaturation with borderline significance $(p=0.06)$, differences in the occurrences of bradycardia and hypotension have not been reported. The pooled occurrence rates of oxygen desaturation, bradycardia, and hypotension in this meta-analysis are reported to be $9.4 \%, 2.8 \%$, and $8.9 \%$, respectively, which are higher than the rates in our study $(2.6 \%, 0.09 \%$, and $0.09 \%$, respectively). Although the reason for the differences is unknown, the studies analyzed in the meta-analysis may have involved invasive procedures such as enteroscopy, endoscopic retrograde cholangiopancreatography, and endoscopic submucosal dissection. In addition, several studies have been conducted to analyze outcomes purely for TCS requiring procedure times longer than those required for EGD and EUS. These invasive or long procedures might have resulted in the use of high dosages, which are related to the occurrence of adverse events.

In their prospective study involving 10,662 EGD examinees, Horiuchi et al. reported the safety of low-dose bolus propofol, for which the maximal dose has been defined to be $120 \mathrm{mg}$, with no adverse events except for the occurrence of a mild decrease in $\mathrm{SpO}_{2}$ of $0.26 \%$ in the patients, requiring transient oxygen administration. ${ }^{8}$ In another prospective single-arm study, they reported administering $0.24 \%$ oxygen for 2,101 TCS examinees, but reported no cardiovascular events after administering a maximal propofol dose of $200 \mathrm{mg} .{ }^{9}$ The low initial dose based on the examinee's age and a maximal dose of $200 \mathrm{mg}$ seem safe to prevent acute unfavorable responses. In our study, although the oxygen desaturation rate was higher (2.6\%) than that in the studies of Horiuchi et al., especially when other drugs were used in combination, no severe events were observed using a similar method. ${ }^{9}$ Our data are obviously favorable in comparison with those obtained from most of the randomized studies with reported incidences of bradycardia (pooled rate, $2.8 \%$; range, $0 \%-6.6 \%)$, hypotension $(8.9 \%$; $0 \%-33.8 \%$ ), and oxygen desaturation (9.4\%; $0 \%-36.8 \%)$.

In the meta-analysis, patient satisfaction was significantly higher in the propofol group $(p<0.001)$ in 6 evaluated studies involving 679 recipients in the settings of outpatient colonoscopy (2 studies from the same institution), ${ }^{10,11}$ outpatient EUS (1 study), ${ }^{12}$ or in-hospital advanced endoscopy such as double-balloon enteroscopy and endoscopic retrograde cholangiopancreatography (the other 3 studies). ${ }^{1,13-15}$ Although no prospective comparative study involving EGD has been conducted, in the single-arm study, Horiuchi et al. ${ }^{8}$ reported that
$94 \%$ of 400 outpatients who underwent EGD reported being satisfied with the sedation for EGD and 99\% wished to be sedated using the same method in future procedures. Considering the results from previous studies and our data from 2,305 examinees, the satisfaction level appears to be acceptable.

None of the participants reported accidents within 24 hours in our study, although car driving was prohibited. In the studies by Horiuchi et al., examinees could drive home after hospital discharge. ${ }^{8,9}$ Of the 400 EGD examinees who replied to the questionnaire, $92 \%$ were reported to have driven without any accidents. ${ }^{8}$ If propofol is proven to have little effect even for relatively risky activities or if the conditions to ensure safety are clarified, propofol sedation would become a more ideal sedation method for endoscopy examinees who desire to do their usual activities during the remainder of the day.

Although our study was not designed to be a comparative study, this is the largest prospective study to have evaluated patient satisfaction for propofol sedation during outpatient endoscopy. Although $4.5 \%$ of the participants desired deeper propofol sedation, $97.7 \%$ hoped to receive propofol sedation in future examinations. Given that this survey was performed using a postal questionnaire after hospital discharge rather than face-to-face interviews, the results seem reliable.

Unfavorable symptoms were evaluated not by the medical personnel but by the examinees, resulting in several reports on symptoms that apparently had no direct relation to sedation, such as throat pain, throat discomfort, abdominal discomfort, and pain at the puncture site for the venous route. These outcomes were impartial evaluations that followed the recent common method to appraise clinical features of drugs. Although these cannot be compared with the outcomes from other examinations or other sedatives, all the symptoms were not significant, and the examinees recovered without medical interventions.

This study has some limitations. First, the design did not include comparative controls such as those who did not receive sedation and those who underwent sedation using another drug. Although randomized designs are theoretically ideal, studies randomizing the use of sedation or kinds of sedatives for screening endoscopy appear ethically difficult to conduct with a large population. Only a single-arm design seems acceptable for prospective evaluation with large population sizes. Second, adverse events were analyzed using only questionnaires without evaluation by medical personnel, resulting in possible overestimations and underestimations, as mentioned earlier. Third, owing to the nature of its prospective design, this study evaluated only participants who completely matched the criteria and provided written informed consent. Some examinees might have experienced some unfavorable events 
but could not submit the questionnaire due to, for example, a serious accident, although the hospital did not receive reports on accidents caused by outpatient sedation during the study period. Fourth, $6.3 \%$ of the participants received sedation using a drug combination, which included diazepam, midazolam, and/or pentazocine. Although these combinations led to the need to administer oxygen (Table 4), no differences were detected in the effect duration, accident occurrence, adverse events, and satisfaction. If the study prohibited the use of a drug combination, the outcome might have been different because a drug combination was used to sedate examinees who could not be effectively sedated using only propofol. However, this prohibition in a protocol is unethical, and a protocol excluding examinees who received a drug combination must involve selection bias. Considering the results of our study, a combination of propofol and other drugs appears to be a reasonable option.

In conclusion, propofol was found to be safe without severe adverse events and accidents for outpatient endoscopy on the basis of next-day patient self-evaluations. Given the high satisfaction level, sedation with propofol might be an ideal tool for painless endoscopic screening.

Conflicts of Interest

The authors have no potential conflicts of interest.

Funding

None.

\author{
Author Contributions \\ Conceptualization: Yoshihide Kanno \\ Data curation: YKa \\ Formal analysis: YKa \\ Investigation: Tetsuya Ohira, Yoshihiro Harada, Shinsuke Koshita, Taka- \\ hisa Ogawa, Hiroaki Kusunose, Yoshiki Koike, Taku Yamagata, Toshita- \\ ka Sakai, Kaori Masu, Keisuke Yonamine, Kazuaki Miyamoto, Megumi \\ Tanaka, Tomohiro Shimada, Fumisato Kozakai, Kazuki Endo, Haruka \\ Okano, Daichi Komabayashi, Takeshi Shimizu, Shohei Suzuki \\ Supervision: Kei Ito \\ Validation: $\mathrm{KI}$ \\ Writing-original draft: YKa \\ Writing-review\&editing: KI
}

\section{ORCID}

Yoshihide Kanno:

Tetsuya Ohira:

Yoshihiro Harada:

Shinsuke Koshita:

Takahisa Ogawa:

Hiroaki Kusunose:

Yoshiki Koike:

Taku Yamagata:

Toshitaka Sakai:

Kaori Masu:

Keisuke Yonamine:
Kazuaki Miyamoto:

Megumi Tanaka:

Tomohiro Shimada:

Fumisato Kozakai:

Kazuki Endo:

Haruka Okano:

Daichi Komabayashi:

Takeshi Shimizu:

Shohei Suzuki:

Kei Ito: https://orcid.org/0000-0001-8313-1396 https://orcid.org/0000-0002-8936-4948 https://orcid.org/0000-0002-8361-0730 https://orcid.org/0000-0002-5890-3531 https://orcid.org/0000-0003-3398-8839 https://orcid.org/0000-0002-0287-1120 https://orcid.org/0000-0003-4202-1574 https://orcid.org/0000-0003-2908-6007 https://orcid.org/0000-0001-6956-2608 https://orcid.org/0000-0002-0023-7919

\section{REFERENCES}

1. Delgado AAA, de Moura DTH, Ribeiro IB, et al. Propofol vs traditional sedatives for sedation in endoscopy: a systematic review and meta-analysis. World J Gastrointest Endosc 2019;11:573-588.

2. Grant SA, Murdoch J, Millar K, Kenny GN. Blood propofol concentration and psychomotor effects on driving skills. Br J Anaesth 2000;85:396-400.

3. Riphaus A, Gstettenbauer T, Frenz MB, Wehrmann T. Quality of psychomotor recovery after propofol sedation for routine endoscopy: a randomized and controlled study. Endoscopy 2006;38:677-683.

4. Goudra B, Nuzat A, Singh PM, Borle A, Carlin A, Gouda G. Association between type of sedation and the adverse events associated with gastrointestinal endoscopy: an analysis of 5 years' data from a tertiary center in the USA. Clin Endosc 2017;50:161-169.

5. Vaessen HH, Knape JT. Considerable variability of procedural sedation and analgesia practices for gastrointestinal endoscopic procedures in Europe. Clin Endosc 2016;49:47-55.

6. Oh JE, Lee HJ, Lee YH. Propofol versus midazolam for sedation during esophagogastroduodenoscopy in children. Clin Endosc 2013;46:368372.

7. Horiuchi A, Nakayama Y, Fujii H, Katsuyama Y, Ohmori S, Tanaka N. Psychomotor recovery and blood propofol level in colonoscopy when using propofol sedation. Gastrointest Endosc 2012;75:506-512.

8. Horiuchi A, Nakayama Y, Hidaka N, Ichise Y, Kajiyama M, Tanaka N. Low-dose propofol sedation for diagnostic esophagogastroduodenoscopy: results in 10,662 adults. Am J Gastroenterol 2009;104:1650-1655.

9. Horiuchi A, Nakayama Y, Kajiyama M, et al. Safety and effectiveness of propofol sedation during and after outpatient colonoscopy. World J Gastroenterol 2012;18:3420-3425.

10. Sipe BW, Rex DK, Latinovich D, et al. Propofol versus midazolam/ meperidine for outpatient colonoscopy: administration by nurses supervised by endoscopists. Gastrointest Endosc 2002;55:815-825.

11. Ulmer BJ, Hansen JJ, Overley CA, et al. Propofol versus midazolam/fentanyl for outpatient colonoscopy: administration by nurses supervised by endoscopists. Clin Gastroenterol Hepatol 2003;1:425-432.

12. Dewitt J, McGreevy K, Sherman S, Imperiale TF. Nurse-administered propofol sedation compared with midazolam and meperidine for EUS: a prospective, randomized trial. Gastrointest Endosc 2008;68:499-509.

13. Vargo JJ, Zuccaro G Jr, Dumot JA, et al. Gastroenterologist-administered propofol versus meperidine and midazolam for advanced upper endoscopy: a prospective, randomized trial. Gastroenterology 2002;123:8-16.

14. Riphaus A, Stergiou N, Wehrmann T. Sedation with propofol for routine ERCP in high-risk octogenarians: a randomized, controlled study. Am J Gastroenterol 2005;100:1957-1963.

15. Schilling D, Rosenbaum A, Schweizer S, Richter H, Rumstadt B. Sedation with propofol for interventional endoscopy by trained nurses in high-risk octogenarians: a prospective, randomized, controlled study. Endoscopy 2009;41:295-298. 